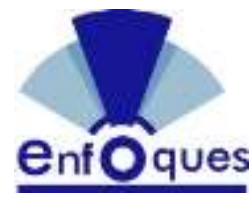

\section{CULTURA ORGANIZACIONAL DE LAS UNIVERSIDADES PÚBLICAS DEL MUNICIPIO CABIMAS}

\author{
ORGANIZATIONAL CULTURE OF THE PUBLIC UNIVERSITIES \\ OF THE MUNICIPALITY CABIMAS
}

ANA CECILIA RIVAS CUICAS

Artículo recibido diciembre 2019 | Arbitrado enero 2020 | Publicado 01 de abril 2020

\begin{abstract}
Resumen
La presente investigación tiene como finalidad describir la cultura organizacional en las universidades públicas del municipio Cabimas. Metodológicamente se caracterizó como una investigación de tipo descriptiva, con un diseño no experimental de campo y transeccional. La población estuvo constituida por dos (2) universidades públicas pertenecientes al municipio Cabimas, de donde se tomó una muestra finita conformada por 69 docentes que desempeñaban cargos administrativos. Para el análisis de la información obtenida se emplearon como técnicas de tratamiento de datos la estadística descriptiva, específicamente la frecuencia absoluta y porcentual. Los resultados de esta investigación indican que el tipo de cultura organizacional que predomina en las instituciones universitarias es la burocrática, porque existe un seguimiento de las reglas de manera metódica, racional y organizada.
\end{abstract}

Palabras clave: Redes sociales; instituciones de educación superior; ocio; amigos; gestión informativa

\begin{abstract}
The purpose of this research is to describe the organizational culture in the public universities of the Cabimas municipality. Methodologically it was characterized as a descriptive type investigation, with a non-experimental field and transectional design. The population was made up of two (2) public universities belonging to the Cabimas municipality, from which a finite sample was taken consisting of 69 teachers who held administrative positions. For the analysis of the information obtained, descriptive statistics were used as data treatment techniques, specifically the absolute and percentage frequency. The results of this research indicate that the type of organizational culture that predominates in university institutions is bureaucratic, because there is a follow-up of the rules in a methodical, rational and organized way.
\end{abstract}

Key words: Social networks; higher education institutions; leisure; friends; information management
Ana Cecilia Rivas Cuicas rivascuicasanacecilia@gmail.com Universidad del Zulia, Venezuela.

Licenciada en Comunicación Social mención Periodismo Impreso. Magister Scientiarum en Ciencias de la Comunicación de la Universidad del Zulia. 


\section{INTRODUCCIÓN}

Hoy día la cultura ha cobrado gran importancia en el ámbito empresarial, puesto que se reconoce que ésta influye, en gran manera, en la forma en cómo funcionan las organizaciones, lo cual se refleja en las estrategias, estructuras y sistemas que implementan con $y$ a través de sus empleados. Asimismo, se ha logrado entender que las culturas fuertes y sólidas no se forman y se mantienen por sí mismas, sino que la organización y los empleados tienen como misión conservar, transmitir e incluso modificar, cuando así lo amerite, dicha cultura.

La cultura de una organización tiene un carácter dinámico, lo cual le permite ser interpretada y reinterpretada, desde su creación, por los empleados en el cumplimiento de sus funciones, en el intento planificado de alcanzar los objetivos que les fueron delegados, generando así subculturas o culturas específicas.

Asimismo, dicha cultura sirve de marco de referencia a los miembros de la organización y da las pautas acerca de cómo las personas deben conducirse en ésta. En muchas ocasiones la cultura es tan evidente que se puede notar que la conducta de la gente cambia en el momento en que traspasa las puertas de la empresa.

Para muchos estudiosos de la administración la cultura organizacional es considerada la base del funcionamiento organizacional, así como en una fuente invisible de energía. En razón de esto, Allaire y Firsirotu, 1992 consideran que para que una empresa lleve a cabo de manera exitosa cualquier tipo de transformación organizacional, es menester, primeramente, generar los cambios en su cultura organizacional, alineándola con las estrategias, las estructuras y los sistemas formales, para lo cual debe contarse con el talento, la disposición y actitud de la gerencia, a objeto de que los miembros de la organización se identifiquen y trabajen en pro de dichos cambios.

Diversos estudios ofrecen evidencia del impacto positivo de la cultura a nivel organizacional. Así, a partir de 1991 Hofstede centra y profundiza sus estudios en el ámbito organizacional y la relación de la cultura con la dirección. A través de estos estudios se enfatiza que si las personas sienten; piensan y actúan de diferentes maneras, es gracias a la cultura. Afirma, además, que la cultura, estructura de maneras diferentes la mente de las personas. Para explicarlo adopta el término "el software de la mente", ya que la cultura programa mentalmente a las personas.

De igual manera, según un informe de Randstand "Work Watch (2010 c.p. Hernández, De la Garza, y Ortiz, 2014) sobre la cultura organizacional, se obtuvieron respuestas significativas, tales como:

...el $66 \%$ de trabajadores encuestados están de acuerdo en que la cultura organizacional es muy importante para el éxito de sus empresas, $35 \%$ que tiene mayor impacto sobre la moral de empleados, $22 \%$ que lo tiene sobre su productividad y $23 \%$ de los más jóvenes (entre 18 y 34 años) creen que lo tiene sobre la satisfacción laboral (p. 68).

En América Latina los estudios realizados sobre la cultura organizacional arrojan resultados que guardan relación con los realizados a nivel internacional. Investigaciones como las de Calderón y Naranjo (2007) quienes han desarrollado varias investigaciones sobre la cultura organizacional de las empresas colombianas y la innovación, con resultados que les lleva a concluir que las empresas innovadoras 
tienen una cultura claramente extrovertido, se focalizan en los clientes y son abiertas al medio ambiente, lo cual reafirma Martínez (2010) cuando considera que las cultura orientadas a lo externo es el único rasgo que incide en el desempeño de las empresas colombianas estudiadas.

En Venezuela, trabajos como los de Ramírez (2010) quien aborda la relación entre la cultura organizacional y las tareas gerenciales del director, confirman la incidencia entre ambas variables, al demostrar que una modificación positiva de la cultura organizacional impactaba de forma positiva sobre las tareas gerenciales del director.

Igualmente, Villegas (2017), en su estudio del impacto de la cultura de una organización del sector farmacéutico sobre la satisfacción laboral de los empleados y determinó que en esta organización la cultura era atomizada en varias subculturas, que privilegiaba el enfoque hacia el presupuesto de ventas y la maximización de las ganancias, más que la atención hacia el capital humano, lo cual impactaba desfavorablemente la satisfacción del personal. Estos hallazgos permiten ratificar la influencia que la cultura organizacional ejerce sobre la satisfacción del personal.

Estos estudios se constituyen en indicadores que confirman el impacto que ejerce la cultura organizacional de una empresa en todos sus empleados y la cual influye no solo en la percepción -moral de los empleados, sino también en el compromiso, la productividad, la salud física, el bienestar emocional. Obviamente este impacto puede ser positivo o negativo, según sea la cultura organizacional presente.

Sin embargo, pese a la importancia de la cultura en las organizaciones, las investigaciones sobre este tópico han estado promovidas, en su mayoría, por el sector empresarial, en tanto que en las organizaciones no empresariales no son tan abundantes. Con respecto a las universidades venezolanas, los estudios relacionados con la cultura organizacional se consideran relativamente nuevos, situación que no pasa desapercibida para la universidad pública del municipio Cabimas.

En razón de esto, las universidades públicas del municipio Cabimas requieren intensificar los estudios respecto a la cultura organizacional, puesto que por ser instituciones cuyos objetivos están direccionados hacia factores medulares como la calidad de la educación, humanización, competencia en la formación de profesionales, por citar algunos, la comprensión de dicha cultura se constituye en parte fundamental del proceso de gestión, lo que permite a sus directivos actuar en forma estratégica y eficiente (Schein, 1984).

De igual manera, las universidades, como parte del proceso evolutivo social, económico y tecnológico de la humanidad, constituyen un marco de valores, creencias, pensamientos e ideales que se ven evidenciados en la cultura organizacional que sirve de referencia para sus miembros en cuanto a comportamiento, identidad y sentido de pertenencia.

Además, resulta determinante para estas instituciones lograr la identificación y difusión correcta de esta serie de valores para provocar cambios en el sentido de pertenencia e identidad de los miembros de una organización y del público externo, que permiten desarrollar una actitud positiva de los empleados hacia la misión, visión y funcionamiento de la institución, cuyo objetivo se vería expuesto en el aumento de los resultados y metas institucionales alcanzados. 
Asimismo, es necesario reorientar la cultura organizacional hacia los propósitos institucionales para que las normas puedan resultar efectivas a medida que los individuos y procesos cambian (Paniagua, Gómez y Fernández 2012). Por otra parte, la cultura además de proporcionar una fuerte cohesión entre sus miembros, debe ser flexible y estimular la motivación, el compromiso y la creatividad hacia los cambios necesarios para la adaptación de la organización a nuevas condiciones y desafíos del entorno.

En razón de lo planteado, surge la necesidad de dar respuesta a la siguiente pregunta de investigación ¿Cuál es el tipo de cultura que presentan las universidades públicas del municipio Cabimas del estado Zulia?

Al hablar de cultura organizacional se hace referencia a un patrón de conducta común utilizado por los individuos y grupos que integran una organización con personalidad y características propias. En razón de esto, se infiere que toda organización tiene una cultura que le permite diferenciarse de las otras empresas, y que de acuerdo al grado de aceptación que posean los miembros puede significar una influencia en las actividades y comportamientos presentes en los empleados.

Ante lo expuesto, Fernández (2007), explica que la cultura juega un papel muy importante dentro de las organizaciones ya que surge como un factor estabilizador en la supervivencia, adaptación y prosperidad cuando se suscitan momentos de turbulencia o transición en las gerencias.

La cultura organizacional, según Chiavenato (2009, p.178), "representa las normas informales y no escritas que orientan el comportamiento de los miembros de una organización en su quehacer diario y que dirige sus acciones para alcanzar sus objetivos".

Asimismo, para Robbins (2009), este concepto enmarca un sistema de significado compartido por los miembros, es la forma como ellos perciben las expectativas de la organización, pero a la vez explica que es difícil de medir su aceptación debido a que es intangible, implícita y, a veces, se da por sentada. A su vez, el autor explica que "cada organización desarrolla un grupo central de suposiciones, conocimientos y reglas implícitas que gobiernan el comportamiento día a día en el lugar de trabajo" y el logro de las metas institucionales.

De igual forma, para Hellriegel y Slocum (2009, p. 458) "la cultura organizacional refleja los valores, creencias y actitudes que han aprendido y comparten sus miembros". Según los autores antes mencionados, este enunciado representa tradiciones que han evolucionado lentamente a lo largo del tiempo y que han surgido como tácticas para incrementar la productividad, lealtad y rentabilidad de las instituciones, a la vez que influyen en los procesos de tomas de decisiones organizacionales.

En este orden de ideas, los autores coinciden en que la cultura organizacional representa los valores, creencias y actitudes de los miembros de la organización, que influyen directamente en el comportamiento de estos en su rutina diaria. Ante esto, el concepto expresado por Robbins (2009) explica un poco mejor la cultura organizacional debido a que refiere que son características claves que la institución valora y que muestra un panorama completo entre lo que deben hacer y cómo deben hacerlo.

Por lo antes expuesto, la cultura organizacional representa una percepción común de la organización y que es utilizada como herramientas para crear, guiar y dirigir 
las actitudes de los empleados hacia el logro de las metas corporativas. Incluye los principios, políticas, procedimientos, valores, esquemas de autoridad y responsabilidad, actitud frente a los cambios tecnológicos, entre otra, lo cual le diferencia de otras instituciones.

En este sentido, la cultura organizacional presente en las universidades engloba una serie de fundamentos constituidos por un sistema de normas, procedimientos $y$ prácticas, en las que se basan el comportamiento de los miembros de su organización para la consecución de los objetivos institucionales enmarcados dentro de los pilares fundamentales de las universidades.

Así pues, la cultura organizacional en las universidades tiene como finalidad contribuir de manera progresiva a fomentar el compromiso y participación de la comunidad universitaria en el accionar diario de la institución, con el firme propósito de favorecer al correcto funcionamiento de los procesos académicos y administrativos.

Cada institución posee su propia cultura organizacional que son factores que la diferencian de las demás; esta separación entre los sistemas compartidos por una y otra empresa, representadas por el comportamiento de los miembros, se percibe por los elementos que conforman la cultura organizacional, los cuales orientan las acciones hacia el logro de los objetivos institucionales.

Igualmente, Robbins (2009) señala que la cultura ayuda a mantener unida a la organización al proporcionar normas adecuadas para las actitudes, estos se evidencian a través de los elementos presentes dentro de la cultura, lo cual, permite identificar y evaluar si estos necesitan ser cambiados o reforzados para adaptarse a los tiempos y momentos que vive la organización o sus miembros.

De la misma manera, Hellriegel y Slocum (2009) expresa que la cultura representa las reglas del juego dentro de una organización, y se desarrolla para darle respuesta al reto de adaptarse al entorno, por lo que utiliza una serie de elementos que en conjunto proyectan el concepto de cultura organizacional y le dan sentido a la presencia de esta.

Consecuentemente, estos autores identifican la presencia de los elementos que conforman la cultura organizacional como ejes de dirección para fortalecer un sistema de representación de la empresa. Según Vargas (2007 p. 132) la cultura organizacional está conformada por "un conjunto de elementos de la vida psíquica y mental, con sus componentes afectivos, cognoscitivos, intelectuales y racionales, que la canalizan como un instrumento para captar a los individuos hacia el logro de metas".

En este orden de ideas, se determina que los elementos de la cultura organizacional son factores que integran los aspectos físicos y espirituales para crear, reforzar y mantener un comportamiento entre los miembros de una institución que les indica que hacer y cuando hacerlo, a la vez, que significan la base para eliminar modelos conceptuales que venían vinculados a los juicios de valor del empleado, construyendo así una visión estratégica. Estos elementos son denominados: simbólicos, estructurales, materiales y conductuales.

Para Robbins (2009), los elementos simbólicos están presentes en todas las organizaciones, estos componen la cultura y expresan la identidad corporativa de la institución, a la vez, que se convierten en 
una representación del sistema compartido por los integrantes de la empresa.

A su vez, Vargas (2007) explica que los símbolos son elementos invisibles que manifiestan significados dentro de una cultura organizacional, ya que a través de manifestaciones verbales, artefactos, ceremonias o rituales, entre otras cosas, se crea la idea previa de un sistema compartido, a partir de las cuales el individuo desarrolla una percepción y de acuerdo a esta, orienta su comportamiento.

Por otro lado, Hellriegel y Slocum (2009 p. 459) determinan que los símbolos son "un conjunto de elementos como lo son las palabras (jerga o modismos), los gestos y las imágenes $u$ otros objetos físicos que tienen un significado particular dentro de una cultura" y a partir de la cual los miembros de una organización generan un comportamiento.

Así pues, los autores coinciden en que los elementos simbólicos de una cultura organizacional son los componentes presentes en la organización y a través de los cuales los miembros desarrollan un significado particular.

En este sentido, los elementos simbólicos, verbales y físicos, representan una percepción particular de un sistema compartido por los individuos la cual permite que se pueda crear una visión y orientar una acción personal hacia la consecución de los objetivos institucionales y personales.

De acuerdo a Vargas (2007, p.164) los elementos estructurales presentes en la cultura organizacional "están relacionados con los sistemas formales desarrollados por la institución, como por ejemplo: niveles de autoridad y decisión, establecimiento de normas y políticas, estrategias, procedimientos, tramos de control $y$ jerarquías".
De igual forma, Hellriegel y Slocum (2009) señalan que las estructuras organizacionales son el conjunto de normas o niveles que conllevan al establecimiento de valores entre los grupos, pues a partir de estas se fomentan y mantienen relaciones laborales.

Asimismo, Robbins (2009) explica que la base estructural de una organización permite moldear la conducta de los miembros, además de determinar las responsabilidades dentro de cada sector de la empresa y que a más niveles de jerarquía se necesitan más elementos que formen la conducta.

Ante lo expuesto por los citados autores se puede evidenciar que los elementos estructurales son componentes importantes de la cultura organizacional, ya que moldean el comportamiento organizacional a través de distribuciones jerárquicas que ayudan a crear responsabilidades y modelos de conducta. Igualmente, Vargas (2007) considera que cada conducta creada por el nivel de rango necesita una serie de valores que establezcan los comportamientos adecuados ante ciertas situaciones laborales.

Así pues, los elementos estructurales podrían ser determinados como componentes organizacionales que utilizan una base organizacional de jerarquía, de normas y valores para generar reglas de conducta para fomentar una mayor flexibilidad y agilidad ante las interacciones necesarias ante el conjunto de significados compartidos entre los miembros.

Según los criterios de Robbins (2009 p. 565) los elementos materiales "transmiten a los empleados quién es importante, el grado de igualdad que desea la alta dirección y los tipos de comportamiento (por ejemplo, de propensión al riesgo, conservador, autoritario, participativo, individualista y social) que son apropiados". 
Por otro lado, Chiavenato (2009) explica que los elementos materiales constituyen aspectos de la comunicación no verbal y que son utilizados para determinar el grado de igualdad o diferencia entre los miembros que conforman la base estructural de la organización.

De la misma forma, Vargas (2007) determina que este tipo de elementos que conforma la cultura organizacional se refieren a materiales físicos como inmobiliarios, equipos o instalaciones que representan o demarcan los niveles de responsabilidad y jerarquía dentro de la institución, por tanto, es posible expresar que los elementos materiales dentro de una cultura organizacional marcan la diferencia entre los sectores laborales de la organización.

Por lo anterior expuesto, se determina que los elementos materiales representan dentro de la organización la separación entre los miembros que conforman la jerarquía institucional y ante la cual se conforma una cultura organizacional a través de un sistema compartido de valores y creencias que repercuten en el comportamiento de los individuos.

A su vez, Robbins (2009), identifica los elementos conductuales, dentro de la cultura organizacional, como aquellos indicadores que fortalecen la identidad corporativa y satisfacción laboral de los miembros que conforman la institución. Igualmente, Vargas (2007) define los elementos conductuales como aquellos que guardan relación con el comportamiento del individuo, tales como como los sistemas de motivación, comunicación, procesos de toma de decisiones, entre otros.

Asimismo, Hellriegel y Slocum (2009) explican que dentro de la cultura organizacional se encuentran diferentes tipos de conductas que reflejan las características internas e individuales de cada uno de los miembros y que influyen en las decisiones institucionales.

Ante lo expuesto, los autores coinciden en que los elementos conductuales son aquellos indicadores que diferencian la cultura organizacional y que determinan la representación de las normas establecidas, además, que refuerzan y ayudan a preservar la cultura, en razón de esto, los elementos conductuales se refieren a las manifestaciones realizadas por los miembros de la organización y, además, representadas a través el comportamiento que indican el nivel de aceptación hacia ciertas normativas.

La cultura organizacional representa los valores, creencias, suposiciones o conocimientos que identifican a una empresa diferenciándola de las otras y a través de la cual sus miembros establecen su comportamiento de rutina en el lugar de trabajo, su manera de pensar, sentir y actuar, en razón de esto, cuando se estudia la cultura organizacional es medular determinar la tipificación de esta con el propósito de entenderla mejor, además de que al tener un conocimiento previo, ante una reingeniería o cambio, se puede reorientar sus elementos para adaptarla al entorno.

Así pues, a lo largo de los años diversos investigadores han enmarcado los tipos de cultura organizacional con varios términos, Chiavenato (2009) determina que existen dos tipos, como lo son la cultura conservadora (aquellas que no se adapta a los cambios y mantienen una rigurosidad) y la cultura adaptable (buscan adaptarse al entorno y están caracterizadas por ser flexibles y orientadas a la innovación).

De igual forma, Kreitner y Kinicky (2005) señalan que existen tres tipos de cultura, las cuales se establecen dependiendo de las interacciones que emanan de los 
trabajadores: constructiva (que estimula a los empleados a que trabajen juntos para fomentar la participación activa entre los miembros para ayudar al desarrollo), pasivadefensiva (donde los encuentros de los empleados deben ser convencionales sin que afecten sus labores) y agresiva-defensiva (donde los individuos son recompensados por la confrontación de las ideas de los demás).

Asimismo, Hellriegel y Slocum (2009) define cuatro tipos de cultura organizacional a partir de su grado de fortaleza y de funcionalidad, que puede servir como punto de partida para analizar las relaciones entre cultura y crisis, dentro de esta clasificación se encuentran: la cultura burocrática, de clan, emprendedora y de mercado.

Por tanto, existen diversos tipos de cultura organizacional de acuerdo a las características de la empresa y que ayudan a dirigir sus esfuerzos hacia el bien común. Para esta investigación se tomaron las aportaciones de Hellriegel y Slocum (2009), ya que permiten vislumbrar aquellas culturas de acuerdo a su funcionalidad y los cuales serán descritos en los apartados siguientes.

De acuerdo a Luna y Pezo (2005) la cultura burocrática es aquella que son inflexibles, buscan realizar las mismas acciones para obtener los mismos resultados y para ello utilizan un control de normas, procedimientos y rutinas, donde la creatividad no es un proceso viable.

Del mismo modo, Fernández (2010) expresa que este tipo de cultura trata de estandarizar el comportamiento organizacional, de tal forma que la repuesta, hacia ciertas situaciones institucionales, sea siempre pronosticable y según las normas que la empresa especifique.

Asimismo, Hellriegel y Slocum (2009) determinan que dentro de una cultura de tipo burocrática las metas están direccionadas a la previsibilidad, eficiencia y la estabilidad de la organización, logrados a través de la formalidad, reglas, procedimientos, normas y sistemas estandarizados.

Así pues, la cultura burocrática según los autores busca regularizar los procesos de trabajo hacia la estabilidad para lograr la eficiencia de los trabajadores, por tanto, para esta investigación se toma lo expresado por Hellriegel y Slocum (2009) debido a que expresan que este tipo de cultura se caracteriza por presentar un seguimiento de las reglas para ser prudente y cuidadoso son aspectos que se valoran de manera metódica, racional y organizada.

En este sentido, la cultura burocrática es aquella que persigue sus objetivos institucionales por medio de procesos, normas y regulaciones estandarizadas para mantener un control del comportamiento de los individuos, con la idea de obtener el mismo resultado, y con el propósito de lograr una estabilidad.

Igualmente, y de acuerdo al trabajo de campo realizado en esta investigación, resulta importante esclarecer que, dentro de las universidades públicas, este tipo de cultura está presente, puesto que el accionar de los procesos se guían por normas y reglas establecidas con anterioridad, los cuales no se pueden violar, porque representan una guía frente a una situación y la posibilidad de darle respuesta enmarcada dentro de los procesos institucionales.

Por su parte, Daft (2007) explica que la cultura de clan se basa en la participación de los empleados de la organización y en la implicación de estos en los procesos, con el fin de liberar la creatividad que les permita responder a las necesidades del entorno, es decir, este tipo de cultura se enfoca en satisfacer a los miembros de la institución para obtener un alto desempeño. 
Por otro lado, Fernández (2010) señala que el enfoque de clan está dirigido a mejorar la parte interna de la organización a través del uso de normas, valores y creencias compartidas hacia el bien común y así lograr enfrentar los cambios externos.

De la misma manera, Hellriegel y Slocum (2009) determinan que dentro de una cultura de clan prevalece un sentido de pertenencia en los miembros, a través de la socialización, y se desarrolla a una lealtad profunda, para fortalecer la toma riesgos e innovaciones, ya que se cree en las potencialidades de cada quien.

Asimismo, los autores coinciden en que la cultura de clan posee como atributos el compromiso personal, la aceptación de ideas, el trabajo en equipo, la participación voluntaria de los miembros en todas las actividades planificadas, la influencia social y la lealtad para el logro de sus objetivos.

Así pues, la cultura de clan invita a los miembros a formar parte de la toma creativa y participativa de decisiones en pro de las metas institucionales, donde los empleados se sienten moralmente obligados a cumplir con la organización pues desarrollan sentimientos de pertenencia hacia ella.

De igual forma, y de acuerdo al trabajo de campo realizado, este tipo de cultura organizacional está presente dentro de las universidades públicas, evidenciándose en algunas actividades gremiales donde se invitan a los miembros de la comunidad universitaria a ser partícipes de la toma de decisiones en pro de una solución clave a los problemas que pueda estar viviendo la universidad.

Por su parte, Fernández (2010 p. 150) define la cultura emprendedora como aquella "que surge en un contexto que exige responder rápidamente a los múltiples cambios sobrevenidos y a tomar decisiones de alto riesgo", en este tipo de cultura se busca estimular y premiar la creatividad.

De la misma manera, Daft (2007) determina a este tipo de cultura como de adaptabilidad debido a que los fundadores fomentan en los miembros la capacidad de detectar y solucionar los problemas para darle respuestas adecuadas a partir del dinamismo e innovación.

Asimismo, Hellriegel y Slocum (2009) expresan que la cultura emprendedora apuesta a la experimentación y a la vanguardia debido a que para el fundador es la iniciativa individual, la flexibilidad y la libertad son alentadas y recompensadas. De igual manera, esta cultura motiva a detectar, interpretar y traducir las señales del medio ambiente en una respuesta.

Ante lo expuesto, se encuentra un punto de concordancia entre los autores, ya que ellos determinan que una cultura emprendedora se basa en los principios de flexibilidad, dinamismo, vanguardia y libertad individual hacia la consecución de los planes. Por ende, la cultura emprendedora crea un ambiente que ánima a los miembros a asumir riesgos con dinamismo y creatividad para estar a la vanguardia e innovación y así responder con inmediatez a los cambios del entorno o a ser generadores de estos.

En este sentido, y de acuerdo al trabajo de campo realizado en esta investigación, la cultura emprendedora se evidencia poco en las instituciones públicas de educación superior, y es representada en algunos procesos, donde la premura o la importancia de la situación lleva al empleado o profesor a dar una respuesta viable, acertada y empática, sin violaciones a las normas, a quienes hacen uso de los servicios brindados por las universidades. 
Para Hellriegel y Slocum (2009 p. 472) la cultura de mercado son relaciones contractuales entre los individuos y la organización, este tipo de cultura "se caracteriza por el logro de los objetivos mensurables y exigentes especialmente aquellos que son financieros y se basan en el mercado".

Igualmente, Fernández (2010) expresa que este tipo de cultura organizacional está dirigida a la obtención de resultados financieros, medibles y exigentes utilizando valores individuales como la competitividad, la responsabilidad y el trabajo arduo para generar un desempeño que sea compensado.

Asimismo, Daft (2005) señala que, en la cultura de mercado, o como él la define "de misión", se posee una visión clara de los objetivos organizacionales y los caminos para el logro de estos utilizando la comunicación y visualización de un panorama para encaminar el comportamiento de los miembros hacia esas metas cuantificables.

En efecto, los autores coinciden en que la cultura de mercado se interesa en obtener ventajas económicas y por ende dirige sus esfuerzos hacia ese fin. En este sentido, la cultura de mercado se basa en los valores de competitividad y de la relación contractual entre los miembros y los directivos donde el valor está determinado por el grado de esfuerzo realizado para la consecución de metas tangibles.

Igualmente, y de acuerdo al trabajo de campo realizado en esta investigación, este tipo de cultura organizacional no se ve reflejado en el accionar de las universidades públicas, ya que a pesar de que algunos procesos administrativos tienen que ser pagados, las instituciones tienen como base primordial la docencia, investigación y extensión como pilares para la consecución del bienestar institucional.

\section{METODOLOGÍA}

La presente investigación se consideró de tipo descriptiva. El diseño fue no experimental de diseño de campo y transeccional. La población correspondiente para esta investigación estuvo constituida por dos (2) universidades públicas pertenecientes al municipio Cabimas, de donde se tomó una muestra finita conformada por 69 docentes, distribuidas de la siguiente manera: 22 pertenecientes a la Universidad del Zulia (LUZ) y 47 pertenecientes a la Universidad Nacional Experimental Rafael María Baralt (UNERMB). La técnica de recolección de datos utilizada fue la encuesta. El instrumento lo constituyó un cuestionario de tipo escala Likert, con opciones múltiples de respuestas y de aplicación autoadministrada. Para su validez fue sometido al escrutinio de 7 expertos. El grado de confiabilidad del instrumento fue determinado a través del Coeficiente de Alfa Crombach, obteniéndose un índice de confiabilidad de 0,87 , que para el caso del instrumento aplicado denotó un rango de muy alta en confiabilidad. Para el análisis de la información obtenida se emplearon como técnicas de tratamiento de datos la estadística descriptiva, específicamente la frecuencia absoluta y porcentual.

En este sentido, para la interpretación de la media se construyó un baremo de medición, donde se expuso, de acuerdo a la alternativa de respuesta con su valor, el intervalo y la categoría a la cual pertenece la puntuación obtenida. 
Tabla 1. Baremo para la interpretación de la media

\begin{tabular}{ccrc}
\hline Rango & Alternativa & Intervalos/Límites & Categoría \\
\hline 5 & Totalmente de acuerdo & $4,21 \geq X \leq 5,00$ & Muy Alta presencia \\
4 & De acuerdo & $3,41 \geq X \leq 4,20$ & Alta presencia \\
3 & Mediano acuerdo & $2,61 \geq X \leq 3,40$ & Mediana presencia \\
2 & En desacuerdo & $1,81 \geq X \leq 2,60$ & Baja presencia \\
1 & Total desacuerdo & $1,00 \geq X \leq 1,80$ & Muy Baja presencia
\end{tabular}

RESULTADOS

Con relación al análisis del indicador burocrática, los resultados mostrados en la tabla 2, reflejaron lo siguiente: $49,28 \%$ de los encuestados estuvieron totalmente de acuerdo en que la cultura burocrática representa la cultura organizacional de las universidades públicas del municipio Cabimas, asimismo, $14,01 \%$ consideraron estar de acuerdo con la presencia de esta cultura en dichas casa de estudios. Un $22,22 \%$ estuvo medianamente de acuerdo; en tanto que $11,59 \%$ y 2,90 estuvieron en desacuerdo y totalmente en desacuerdo, respectivamente, al considerar que la burocrática es el tipo de cultura organizacional de las universidades objeto de este estudio.

De igual manera puede notarse que la media para este indicador fue de 4,03, ubicándose en la categoría de muy alta presencia, lo cual implica que en las universidades públicas del municipio Cabimas la cultura burocrática tiene una alta presencia.

Con respecto al indicador denominado de clan, $12,56 \%$ de la población identificó estar totalmente de acuerdo con que este tipo representa la cultura organizacional de las instituciones antes señaladas; $8,70 \%$ consideraron estar de acuerdo; de igual forma $34,78 \%$ afirmó estar medianamente de acuerdo en que la cultura de clan es la que enmarca a dichas casas de estudio; en tanto que, $31,88 \%$ estuvo en desacuerdo frente a $12,08 \%$ que afirmó estar totalmente en desacuerdo. La media para este tipo de indicador fue de 2,78 o cual implica la existencia de una mediana presencia de la cultura de clan es estas instituciones. (Tabla 2).

Variable: Cultura Organizacional

Dimensión: Tipo de cultura organizacional 
Tabla 2. Tipos de Cultura Organizacional

\begin{tabular}{ccccccccc}
\hline \multicolumn{7}{c}{ Dimensión: Tipos de Cultura Organizacional } \\
\hline Indicador & \multicolumn{2}{c}{ Burocrática } & \multicolumn{3}{c}{ De Clan } & Emprendedora & De Mercado \\
\hline Alternativas & FA & FR & FA & FR & FA & FR & FA & FR \\
TA & 34 & $49,28 \%$ & 9 & $12,56 \%$ & 1 & $1,93 \%$ & 1 & $0,97 \%$ \\
A & 10 & $14,01 \%$ & 6 & $8,70 \%$ & 13 & $19,32 \%$ & 8 & $11,11 \%$ \\
MA & 15 & $22,22 \%$ & 24 & $34,78 \%$ & 23 & $32,85 \%$ & 18 & $25,60 \%$ \\
D & 8 & $11,59 \%$ & 22 & $31,88 \%$ & 24 & $34,30 \%$ & 28 & $41,06 \%$ \\
TD & 2 & $2,90 \%$ & 8 & $12,08 \%$ & 8 & $11,59 \%$ & 15 & $21,26 \%$ \\
TOTAL & 69 & $100 \%$ & 69 & $100 \%$ & 69 & $100 \%$ & 69 & $100 \%$ \\
\hline Media Indicador & & 4,03 & & 2,78 & & 2,66 & & 2,29 \\
\hline
\end{tabular}

Para el análisis del indicador denominado emprendedora, los resultados arrojados fueron los detallados a continuación: $1,93 \%$ de los encuestados afirman estar totalmente de acuerdo de que este tipo de cultura organizacional está presente en las casas de estudios del municipio Cabimas, mientras, 19,32\% consideró estar de acuerdo. Otro 32,85\% estuvo medianamente de acuerdo; a la vez, que el $34,30 \%$ en desacuerdo, mientras que un $11,59 \%$ afirmó estar totalmente en desacuerdo que la cultura emprendedora represente a los miembros de la comunidad universitaria. La media obtenida en el análisis de este indicador fue de 2,66. (Tabla 2).

De igual forma, para el indicador identificado como de mercado, 0,97\% de la población estuvo totalmente de acuerdo con que este tipo de cultura organizacional representaba a las universidades públicas, en tanto que un $11,11 \%$ consideró estar de acuerdo; de igual forma 25,60 \% afirmó estar medianamente de acuerdo, en tanto que un $41,06 \%$ respondió estar en desacuerdo frente a $21,26 \%$ que respondió estar totalmente en desacuerdo que la cultura de mercado tenga una presencia en las casas de estudios. La media para este indicador fue de 2,29 .
Así pues, de acuerdo al análisis cuantitativo de los datos, estratificado y presentado por cada indicador dispuestos para medir la dimensión tipos de cultura corporativa, se evidencia que la burocrática predomina en las universidades públicas del municipio Cabimas, ya que presenta un seguimiento de las reglas, la organización es considerada un lugar formalizado y estructurado para trabajar, los procedimientos rigen el quehacer de los trabajadores.

Las reglas, políticas y normas se constituyen en elementos claves para mantener el control de la organización.

El interés de los líderes de estas organizaciones está centrado ser buenos coordinadores y organizadores a fin de mantener la organización cohesionada.

En este sentido, los resultados antes señalados coinciden con lo establecido por Hellriegel y Slocum (2009) quienes consideran que dentro de una cultura de tipo burocrática las metas están direccionadas a la previsibilidad, eficiencia y la estabilidad de la organización, logrados a través de la formalidad, reglas, procedimientos, normas y sistemas estandarizados. 
En efecto, la cultura de tipo burocrática es la que se encuentra presente dentro de las instituciones de educación superior objeto de este estudio, puesto que el accionar de los procesos se guían por normas y reglas establecidas con anterioridad, los cuales no se pueden violar, puesto que representan una guía frente a una situación determinada y la posibilidad de darle respuesta enmarcada dentro de los procesos institucionales.

\section{CONCLUSIONES}

Con respecto a los tipos de cultura organizacional objeto de este estudio, se pudo comprobar que la cultura burocrática es la que se manifiesta en las universidades públicas del municipio Cabimas, puesto que predominan y se siguen las reglas de manera metódica, racional y organizada. Los conflictos que se hacen presentes tienen soluciones previstas y cada trabajo posee su formalidad, reglas, procedimientos, normas y sistemas estandarizados.

Asimismo, para el indicador cultura de clan se evidenció que está poco presente en estas casas de estudio, pues son escasos los escenarios donde se invitan a los miembros a ser parte de la toma de decisiones, no se manifiesta un alto compromiso de sus miembros por las instituciones. Iguales resultados se muestran para la cultura emprendedora y la de mercado, por lo que se puede inferir que no forman parte de las acciones que las universidades quieren compartir con sus miembros en su sistema de asociación.

\section{REFERENCIAS}

Allaire, Y. y Firsirotu, M. (1992). Revoluciones culturales en las grandes organizaciones. En Abravanel y otros Cultura organizacional en Legis. Bogotá, 1992
Calderón, G. y Naranjo, J. (2007). Perfil cultural de las empresas innovadoras: Un estudio de caso en empresas metalmecánicas. Cuadernos de Administración. Javeriana

Chiavenato, I. (2009). Gestión del Talento Humano. México. Editorial Mc Graw Hill

Daft, R. (2007). Teoría y Diseño Organizacional. México. Editorial Cengage Learning

Fernández, M. (2007). Empresas de Rosario: cambio profundo a partir de un modelo de aprendizaje. Rosario: Universidad Nacional de Rosario, Tesis Doctorado en Administración. 267 pp

Hellriegel, D. y Slocum, J. (2009). Comportamiento Organizacional. México: International Thomson Editores

Hernández, N., De la Garza, E., y Ortiz, A. (2014). Generación de Capital Social a partir de la eficacia, la cultura y el aprendizaje organizacional: un estudio correlacional. Revista Internacional Administración y Finanzas

Kreitner, R. y Kinicky, A (2005). Comportamiento de las organizaciones Madrid, España. Editorial Mc Graw-Hill

Luna, R. y Pezo, A. (2005). Cultura de la innovación y la gestión tecnológica para el desarrollo de los pueblos y desarrollo productivo. Bogotá, Colombia. Convenio Andrés Bello

Martínez, M. (2010). Relaciones entre cultura y desempeño organizacional en una muestra de empresas colombianas: reflexiones sobre la utilización del modelo de Denison. Cuadernos de Administración 23(40):163-190 Javeriana

Paniagua, F. y Gómez, B. (2012). Hacia la comunicación 2.0: El uso de las redes sociales por parte de las universidades españolas. Revista ICONO 14 de Comunicación y Nuevas Tecnologías. (Revista en línea) Año 10, №3. Disponible en: http://www.icono14.net/ojs/index.php/i cono14/article/view/473/397 (Consulta 2017, mayo 30) 
Ramírez, J. (2010). Cultura Organizacional y Tareas Gerenciales del Director. Tesis de Maestría (Maestría en Educación Mención: Planificación Educativa) Universidad del Zulia. Maracaibo, edo. Zulia/ Venezuela

Robbins, S. (2009). Comportamiento Organizacional. México: Pearson Educación Editores

Schein, E. (1984). Comong to a new awareness of organizational culture. Sloan mangement review, 25, pp 3-16

Vargas, J. (2007). La culturocracia organizacional en México. México.
Edición electrónica gratuita. Disponible en: www.eumed.net/libros/2007b/

Villegas, C. (2017). Impacto de la cultura organizacional sobre la satisfacción laboral. Caso empresa farmacéutica ubicada en Guacara estado Carabobo. Universidad de Carabobo. Facultad de Ciencias Económicas y Sociales. http://mriuc.bc.uc.edu.ve/bitstream/han dle/123456789/4319/cvillegas.pdf?sequ ence $=1$ 